\title{
Economic Reliability-Aware MPC-LPV based on Chance-Constraints Programming for Water Networks*
}

\author{
Fatemeh Karimi Pour ${ }^{1}$, Vicenç Puig ${ }^{1}$ and Gabriela Cembrano ${ }^{1}$
}

\begin{abstract}
This paper proposed an economic reliabilityaware model predictive control (MPC) for the management of drinking water transport networks (DWNs) that includes a new goal to increase the system and components reliability based on a finite horizon stochastic optimization problem with joint probabilistic (chance) constraints. The proposed approach is based on a single-layer economic optimization problem with dynamic constraints. The inclusion of components and system reliability in the MPC model using an LPV modelling approach aims at maximizing the availability of the system by estimating system reliability. The solution of the optimization problem related to the MPC problem is obtained by solving a series of Quadratic Programming (QP) problem. The use of chanceconstraint programming allows computing an optimal policy based on a desirable risk acceptability level and managing dynamically volume tank stocks to cope with non-stationary flow demands. Finally, the proposed approach is applied to a part of a real drinking water transport network of Barcelona for demonstrating the performance of the method.
\end{abstract}

\section{INTRODUCTION}

Drinking Water Networks (DWNs) are critical infrastructures in urban environments. DWNs are multivariate dynamic constrained systems that are described by the interconnection of several subsystems (tanks, actuators, sources, nodes and consumer demands). Moreover, its optimal management, that can be formulated as a multi-criteria optimization problem, is a complex challenge for water suppliers. To deal with these complexity, Model Predictive Control (MPC) has been established as a suitable method [1]. Regarding optimized control strategies for managing water systems, MPC is not implemented in a classical way because there is no reference to be tracked [2]. For this purpose, Economic MPC (EMPC) provides a systematic method for optimizing economic performance. Latterly, system reliability in the process of control system has been considered through a Prognosis and Health Management (PHM) framework. On the other side, MPC predicts the suitable control actions to obtain optimal performance according to multi-objective cost functions and physical constraints. This is the reason to be considered as a suitable approach for developing health-aware control schemes. The system reliability has a strong relationship with the control input determined by the controller. Several studies on the DWN control are focused on the application of MPC to provide optimal management of the DWN and

\footnotetext{
*This work has been funded by SMART Project (ref. num. EFA153/16 Interreg Cooperation Program POCTEFA 2014-2020).

1 F. Karimi Pour, V. Puig and G. Cembrano are with the Advanced Control Systems Group, Universitat Politècnica de Catalunya, Institut de Robòtica i Informàtica Industrial (CSIC-UPC), C/. Llorens i Artigas 4-6, 08028 Barcelona, Spain. vicenc.puig@upc.edu
}

to satisfy the consumer demand while preserving the DWN reliability, e.g. [3]. However, considering the reliability at the actuator level not at the system level is the main drawback of the previous methods. Otherwise, it leads to the use of non-linear MPC because non-linearity of the resulting constraints. Generally, Economic Nonlinear MPC (ENMPC) is computationally costly and, there is no guarantee that the solution of the optimization problem corresponds to the global optimum. One way to deal with nonlinearity is to represent the process behavior by means of Linear Parameter Varying (LPV) models. Another weakness of previous approaches according to the reliability analysis and MPC strategies is the conservatism of the resultant control policy that effects negatively on the efficiency function of the DWNs operation. A more realistic qualification of uncertainty is the stochastic paradigm. Powerful tools for control design, heading to the Stochastic MPC, which has a particular alternative called Chance-Constrained MPC (CCMPC) [4]. By placing this value suitably, the user/operator can obtained the desired trade-off between robustness and performance. Some economic-oriented controller that consider the reliability issue has been proposed [3], but without considering reliability at the system level and probabilistic constraints based on the reliability of the system.

The aim of this paper is to propose an economic healthaware MPC controller based on PHM information that is provided by the online evaluation of the system reliability according to the probabilistic constraints for the reliability of the system by means of the chance constraints programming paradigm. By using the LPV structure, the system reliability is incorporated into the control algorithm. The augmented model that includes both the reliability models and DWN is expressed as an LPV model that at each time instant the varying parameters are updated according to the value of the scheduling variables. Therefore, the control inputs are determined to satisfy the control objectives and simultaneous to increase the lifespan and reliability of the system components. The use of chance constraints to compute an optimal based on a desired risk acceptability level, system reliability and presenting the advantage of a given system and component reliability that is computed on-line in an MPCLPV strategy is the main goal of this paper.

\section{EMPC FOR TRANSPORT WATER NETWORKS}

\section{A. Control-oriented model}

In this paper, since the transportation layer is considered, a control-oriented modeling approach that is based on a flow model is considered that follows the principles introduced by the authors in [5]. Following flow/mass balance principles, 
a discrete-time model based on linear differential algebraic equations (DAEs) for all time instant $k \in \mathbb{Z}_{>0}$ can be formulated for a given DWN as follows:

$$
\begin{aligned}
x(k+1) & =A x(k)+B u(k)+B_{d} d_{m}(k), \\
0 & =E_{u} u(k)+E_{d} d_{m}(k),
\end{aligned}
$$

where state vector $x \in \mathbb{R}^{n_{x}}$ is defined to represent the tank volumes. The vector $u \in \mathbb{R}^{n_{u}}$ of controlled inputs is associated to the flow rates through the actuators (pumps and valves) of the network, and the vector $d_{m} \in \mathbb{R}^{n_{d}}$ demanded flow as measured disturbances. $A \in \mathbb{R}^{n_{x} \times n_{x}}, B \in$ $\mathbb{R}^{n_{x} \times n_{u}}, B_{d} \in \mathbb{R}^{n_{x} \times n_{d}}, E_{u} \in \mathbb{R}^{n_{d} \times n_{u}}, E_{d} \in \mathbb{R}^{n_{d} \times n_{d}}$ and $C \in \mathbb{R}^{n_{y} \times n_{x}}$ are time-invariant matrices of that depends on the network topology. The system is subject to hard input and state as

$$
\begin{array}{r}
x(k) \in \mathbb{X}:=\left\{x \in \mathbb{R}^{n_{x}} \mid G x \leq g\right\}, \\
u(k) \in \mathbb{U}:=\left\{u \in \mathbb{R}^{n_{u}} \mid H u \leq h\right\},
\end{array}
$$

for all $k \in \mathbb{Z}_{\geq 0}$, where $G \in \mathbb{R}^{m_{x} \times n_{x}}, g \in \mathbb{R}^{m_{x}}, H \in$ $\mathbb{R}^{m_{u} \times n_{u}}$ and $h \in \mathbb{R}^{m_{u}}$ are vectors/matrices collecting the system constraints, signifying $m_{u} \in \mathbb{Z}_{\geq 0}$ and $m_{x} \in \mathbb{Z}_{\geq 0}$ the number of input and state constraints, respectively.

\section{B. EMPC formulation}

The control goal can be formulated as the minimization of a convex multi-objective cost function considering

1) Economic costs Minimization : Minimizing the economical costs that include water production and electrical costs related to pumping is the main control objective of the DWN. Therefore, the cost function related to this objective can be expressed as

$$
\ell_{e}(k) \triangleq \alpha(k)^{\top} W_{e} u(k)
$$

where $\alpha(k) \triangleq\left(\alpha_{1}+\alpha_{2}(k)\right) \in \mathbb{R}^{n_{u}}, \alpha_{1} \in \mathbb{R}^{n_{u}}$ denotes a fixed water-production costs that related to the water treatments and $\alpha_{2} \in \mathbb{R}^{n_{u}}$ corresponds to a time-varying water cost associated to pumping that varies in each time instant $k$ with respect to the dynamic electricity tariff. $W_{e}$ indicates the weighting term.

2) Safety Management: Regarding preserving water stock despite the change of water demands among two consecutive MPC iterations, an appropriate safety capacity for each storage tank is required to be maintained. The safety cost function can be expressed through a soft constraint by adding a slack variable $\xi$ that can be formulated as

$$
\ell_{s}(k) \triangleq \xi^{\top}(k) W_{s} \xi(k),
$$

where the next soft constraint is included $x(k) \geq x_{s}-\xi(k)$ and $x_{s}$ indicates the vector of the safety levels for all the tanks. $W_{s}$ is diagonal positive definite matrix.

3) Smoothness of Control Actions: For guarantee the smoothing effect, the slew rate of the control actions among two sequential time instants is penalized according to

$$
\ell_{\Delta u}(k) \triangleq \Delta u(k)^{\top} W_{\Delta u} \Delta u(k),
$$

where $\Delta u(k) \triangleq u(k)-u(k-1)$, and $W_{\Delta u}$ is a diagonal positive definite matrix.
According to the network model (1), the MPC controller design is based on minimizing the following cost function in the prediction horizon $N_{p}$

$$
J=\sum_{l=0}^{N_{p}}\left(\ell_{e}(l \mid k)+\ell_{s}(l \mid k)+\ell_{\Delta u}(l \mid k)\right) .
$$

where at each time instant, the following optimization problem is solved online.

$$
\min _{\mathbf{u}(k), \mathbf{x}(k), \boldsymbol{\xi}(k)} J(\mathbf{u}(k), \mathbf{x}(k), \boldsymbol{\xi}(k)),
$$

subject to:

$$
\begin{aligned}
& x(l+1 \mid k)=A x(l \mid k)+B u(l \mid k)+B_{d} d_{m}(l \mid k), \\
& 0=E_{u} u(l \mid k)+E_{d} d_{m}(k), l=0, \cdots, N_{p}-1 \\
& x(l \mid k) \geq x_{s}-\xi(l \mid k), l=1, \cdots, N_{p} \\
& u(l \mid k) \in \mathbb{U}, \quad x(l \mid k), \in \mathbb{X} \\
& \xi(l \mid k) \geq 0, \quad x(0 \mid k)=x(k) .
\end{aligned}
$$

The optimal sequences $\mathbf{u}^{*}(k)=\{u(l \mid k)\}_{l \in \mathbb{Z}_{\left[0, N_{p}-1\right]}}$, $\mathbf{x}^{*}(k)=\{x(l \mid k)\}_{l \in \mathbb{Z}_{\left[1, N_{p}\right]}}$ and $\boldsymbol{\xi}^{*}(k)=\left\{x_{i}(l \mid k)\right\}_{l \in \mathbb{Z}_{\left[1, N_{p}\right]}}$ are obtained online. Considering the receding horizon philosophy [1], the procedure is based on solving the optimization problem (7a) from the current time instant $k$ to $k+N_{p}$ by using $x(0 \mid k)$ as the initial condition that is computed from state estimation at time $k$.

\section{Chance-Constrained Model Predictive CONTROL}

If the stochastic nature of disturbances (demands) and reliability of components of the system is not explicitly considered, an finding optimal solution of (7) satisfying all constraints can not be found in real scenarios. Therefore, in order to guarantee feasibiliy of the optimization problem (7), it is appropriate to relax the original constraints that involve stochastic elements with probabilistic statements in the form of chance constraints. Chance-constrained programming is a technique of stochastic programming dealing with constraints of the general form as

$$
\mathbb{P}[\mathrm{f}(v, \zeta) \leq 0] \geq 1-\delta_{\zeta},
$$

where $\mathbb{P}$ indicates the probability operator, $v \in \mathbb{R}^{n_{v}}$ is the decision vector, $\zeta \in \mathbb{R}^{n_{\zeta}}$ a random variable and $\mathrm{f}$ : $\mathbb{R}^{n_{v}} \times \mathbb{R}^{n_{\zeta}} \longrightarrow \mathbb{R}^{n_{c}}$ a constraint mapping. The level $\delta_{\zeta} \in(0,1)$ is user given and defines the preference for safety of the decision $v$. The constraint (8) means that we wish to take a decision $v$ that satisfies the $n_{c}$-dimensional random inequality system $\mathrm{f}(v, \zeta) \geq 0$ with high enough probability. As demonstrated in [6], if $\mathrm{f}(.,$.$) is jointly convex$ in $(v, \zeta)$ and $\Phi \triangleq \mathbb{P}[$.$] is quasi-concave, then the feasible$ set $\Psi\left(\delta_{\zeta}\right) \triangleq\left\{v \mid \mathbb{P}[\mathrm{f}(v, \zeta) \leq 0] \geq 1-\delta_{\zeta}\right\}$ is convex for all $\delta_{\zeta}(0,1)$. All chance-constrained models need prior knowledge of the acceptable risk $\delta_{\zeta}$ connected with the constraints. In this paper, by following the results in [7], a uniform distribution of the joint risk is approximated by upper bounding the joint constraint and assuming a similar distribution of the joint risk amongst a set of individual chance constraints are transformed inside equivalent deterministic constraints. 
By considering the general joint chance constraint (8), and define $\mathrm{f}(v, \zeta) \triangleq \zeta-F v$, with $F \in \mathbb{R}^{\zeta \times n_{v}}$. Therefore, the additive stochastic element is separable and the following chance constraint is achieved:

$$
\mathbb{P}[\zeta \leq F v] \geq 1-\delta_{\zeta}
$$

Then, by rewriting $\omega \triangleq F v$, for any duple $(\zeta, \omega)$, it follows $\Phi_{\zeta}(\omega)=\mathbb{P}\left[\left\{\zeta_{1} \leq \omega_{1}, \ldots, \zeta_{n_{c}} \leq \omega_{n_{c}}\right\}\right]$. Describing the events $C_{i} \triangleq\left\{\zeta_{i} \leq \omega_{i}\right\}, \forall i \in \mathbb{Z}_{1}^{n_{c}}$ (as e.g. faults in the actuators or unexpected changes in the demand), it follows the $\Phi_{\zeta}(\omega)=$ $\mathbb{P}\left[C_{i} \cap \ldots \cap C_{n_{c}}\right]$. Indicating the complements of the events $C_{i}$ by $C_{i}^{c} \triangleq\left\{\zeta_{i}>\omega_{i}\right\}$, and it is obvious from probability theory, $\left(C_{1} \cap \ldots \cap C_{n}=\left(C_{1}^{c} \cup \ldots \cup C_{n_{c}}^{c}\right)^{c}\right)$, consequently

$$
\begin{aligned}
\Phi_{\zeta}(\omega) & =\mathbb{P}\left[C_{i} \cap \ldots \cap C_{n_{c}}\right] \\
& =\mathbb{P}\left[\left(C_{1}^{c} \cup \ldots \cup C_{n_{c}}^{c}\right)^{c}\right] \\
& =1-\mathbb{P}\left[\left(C_{1}^{c} \cup \ldots \cup C_{n_{c}}^{c}\right)^{c}\right] \leq 1-\delta_{\zeta} .
\end{aligned}
$$

By using the union bound, the Boole inequality let to bound the result in (10c), declaring that for a countable set of events, the probability that at least one event occurs is not higher than the sum of the individual probabilities [8], such that

$$
\mathbb{P}\left[\cup_{i=1}^{n_{c}} C_{i}\right] \leq \sum_{i=1}^{n_{c}} \mathbb{P}\left[C_{i}\right],
$$

and, by applying (11) to (10c), it yields to

$$
\sum_{i=1}^{n_{c}} \mathbb{P}\left[C_{i}^{c}\right] \leq \delta_{\zeta} \Longleftrightarrow \sum_{i=1}^{n_{c}}\left(1-\mathbb{P}\left[C_{i}\right]\right) \leq \delta_{\zeta} .
$$

Then, a set of constraints rises from previous results as sufficient conditions to enforce the joint chance constraint (9), by allotting the joint risk $\delta_{\zeta}$ in $n_{c}$ separate risks $\delta_{\zeta, i}, i \in$ $\mathbb{Z}_{i}^{n_{c}}$. These constraints are described as follows

$$
\begin{gathered}
\mathbb{P}\left[C_{i}\right] \geq 1-\delta_{\zeta, i}, \quad \forall i \in \mathbb{Z}_{i}^{n_{c}} \\
\sum_{i=1}^{n_{c}} \delta_{\zeta, i} \leq \delta_{\zeta}, \quad 0 \leq \delta_{\zeta, i} \leq 1
\end{gathered}
$$

where (13a) produces the set of $n_{c}$ effective individual chance constraints. Moreover, (13b) is condition forced to bound the new single risks in such a way that the joint risk bound is not breached. Each solution that satisfies the aforesaid constraints is guaranteed to provide (9).

According to that the satisfaction of each individual constraint is an event $C_{i}, \forall i \in \mathbb{Z}_{i}^{n_{c}}$. A joint chance constraint needs that the connection of all the individual constraints is satisfied with the wanted probability level as $\mathbb{P}\left[\cap_{i=1}^{n_{c}}\right.$ $\left.C_{i}\right] \geq 1-\delta_{\zeta}$. Considering that each individual constraint is probabilistically dependent, the level of conservatism can be derived by using the inclusion-exclusion principle for the union of finite events, $C_{i}, \forall i \in \mathbb{Z}_{1}^{n_{c}}$, which proves the following equality:

$$
\begin{aligned}
\mathbb{P}\left[\cup_{i=1}^{n_{c}} C_{i}\right]= & \sum_{i=1}^{n_{c}} \mathbb{P}\left[C_{i}\right]-\sum_{1 \leq i<j \leq n_{c}} \mathbb{P}\left[C_{i} \cap C_{j}\right] \\
& +\sum_{1 \leq i<j<k \leq n_{c}} \mathbb{P}\left[C_{i} \cap C_{j} \cap C_{k}\right]-\ldots \\
& +(-1)^{n_{c}-1} \mathbb{P}\left[\cap_{i=1}^{n_{c}} C_{i}\right] .
\end{aligned}
$$

It should be noted that by considering as an event a fault in an actuator, it can be observed (14) has a similar as formulation as the one used for evaluating the system reliability based on the component reliability. In a DWN, the constraints come from the model (7b)-(7c) that can be formulated as chance constraints statements taking into account the probabilities associated to the component reliability. Considering only faults in actuators, the reliability of the system is related to the system inputs $u_{i}(k)$. Hence, (8) can be formulated in case of the actuators as follows

$$
\mathbb{P}\left[\mathrm{f}\left(u_{i}(k), \zeta_{i}(k)\right) \leq 0\right] \geq 1-\delta_{\zeta_{i}},
$$

where $\zeta(k) \in\{0,1\}$ is a stochastic variable which considers if the actuator is one of two states $\{$ Unvailable, Available $\}$ (or $\{0,1\}$ ) defined as follows:

$$
\zeta_{i}(k)= \begin{cases}1, & R_{i}(k)>0 \\ 0, & R_{i}(k)=0\end{cases}
$$

where $R_{i}(k)$ is the actuator reliability. In case that $\zeta_{i}(k)=1$ the input $u_{i}(k)$ associated to the $i$-th actuator is bounded by (2b), otherwise an additional constraint setting $u_{i}(k)=0$ should be included. Furthermore, to determine the reliability associated to the system that associates a probability to the system model constraint (1), the joint-chance constraint probability calculation (14) should be used leading to the following probabilistic formulation for the MPC optimization problem (7)

$$
\min _{\mathbf{u}(k), \mathbf{x}(k), \boldsymbol{\xi}(k)} J(\mathbf{u}(k), \mathbf{x}(k), \boldsymbol{\xi}(k)),
$$

subject to:

$$
\begin{aligned}
& \mathbb{P}\left[A x(l \mid k)+B\left(\zeta_{i}\right) u(l \mid k)+B_{d} d_{m}(l \mid k),\right. \\
& \left.\quad E_{u}\left(\zeta_{i}\right) u(l \mid k)+E_{d} d_{m}(k)\right] \geq 1-\delta, \quad l=0, \cdots, N_{p}-1 \\
& \quad x(l \mid k) \geq x_{s}-\xi(l \mid k), \quad l=0, \cdots, N_{p} \\
& \quad u(l \mid k) \in \mathbb{U}, \quad x(l \mid k), \in \mathbb{X} \\
& \quad \xi(l \mid k) \geq 0, \quad x(0 \mid k)=x(k) .
\end{aligned}
$$

The main difficulty in solving this stochastic problem using chance constraints is that at each time iteration, the probabilities associated to the system reliability should be updated taking into account the value of the optimal control actions $u_{i}$.

\section{Augmenting network Model With the RELIABILITY MODEL}

\section{A. Reliability Model}

The general explanation of failure rate, indicated by $\lambda$ is presented as the fraction of the density of the stochastic lifetime to the remainder function (i.e., conditional probability). In this paper, failure rates are determined from actuators under various levels of load respect to the applied control input. The regular used relationship is based on assuming that actuator fault rates varied through the load by the following exponential law

$$
\lambda_{i}=\lambda_{i}^{0} \exp \left(\beta_{i} u_{i}(k)\right), \quad i=1,2, \ldots, m
$$


where $\lambda_{i}^{0}$ represents the baseline failure rate (nominal failure rate) and $u_{i}(k)$ is the control action a time $k$ for the $i$ th actuator. $\beta_{i}$ is a constant parameter that depends on the actuator characteristics. Reliability $R(t)$ is the probability that a system will be successful in the intervening period from time 0 to time $t$. Besides, The unreliability $F(t)$ is determined as the probability that the component or system encounters the first failure or has failed one or more times among the time interval 0 to time $t$. Considering the system (or component) is always in one of the two states introduced in (16), relationship $F(t)+R(t)=1$ is provided.

In the useful life period, the component can be specified at a certain time $t$ by a starting point reliability measure $R_{0}(t)$. Accordingly, $R_{0, i}(t)$ will denote the reliability of the $i$-th actuator determined under nominal operating conditions

$$
R_{0, i}(t)=\exp \left(-\lambda_{i}^{0} t\right), \quad i=1,2, \ldots, m
$$

Hence, the components reliability of a system with the $i$-th components in discrete-time can be computed by exploiting the exponential function and the baseline reliability level $R_{0, j}$ as follows

$R_{i}(k+1)=R_{0, i}(k)+\exp \left(-T_{s} \sum_{s=0}^{k+1} \lambda_{i}(s)\right), \quad i=1,2, \ldots, m$

where $\lambda_{i}(s)$ is the failure rate that is acquired from the $i$ th component under varying levels of load $u_{i}$ and $T_{s}$ is the sampling time.

\section{B. Overall Reliability}

The lifespan of a system can be determined by the overall system reliability that indicated as $R_{g}(k)$. Therefore, $R_{g}(k)$ is influenced by the configuration of the actuator that can generally be computed from parallel and/or series combinations of subsystems (or components) [9]. To manage the more complex situation, a graph network model can be used which by determining the existence of a successful path in the system it can be identified the system whether is working correctly. A minimal path $P_{s}$ is a set of components that relates to it, but the elimination any one of the components will create the set not to be a successful path [9]. Therefore, the overall system reliability $R_{g}(k)$ can be counted as

$$
R_{g}(k)=1-\prod_{j=1}^{s}\left(1-\prod_{i \in P_{s, j}} R_{i}(k)\right)
$$

where $j=1, \ldots, s$ is minimal paths number. As mentioned in previous section, there is indirect relationship between conservatism of probability and the overall system reliability. In fact, the formula obtained for overall reliability system (21) can be obtained from (14).

\section{ECONOMIC RELIABILITY-AWARE MPC-LPV USING CHANCE-CONSTRAINTS}

\section{A. Economic reliability aware $M P C-L P V$}

In this section, the integration of reliability model in the MPC controller augmenting the DWN model is proposed. A new objective can be included in the MPC controller that aims to preserve the system reliability additionally to consider the reliability model (21). The augmented MPC model can be formulated as follows

$$
\begin{aligned}
x_{g}(k+1) & =A_{g} x_{r}(k)+B_{g} u(k)+B_{d, g} d_{m}(k), \\
y_{g}(k) & =C_{g} x(k),
\end{aligned}
$$

where the state and output vector are given by $x_{g}=$ $\left[x, \log \left(Q_{g}\right), \log \left(R_{1}\right), \ldots, \log \left(R_{i}\right)\right]^{T}$ and $y_{g}=\left[y, \log \left(Q_{g}\right)\right]^{T}$, respectively. The augmented matrices are defined as

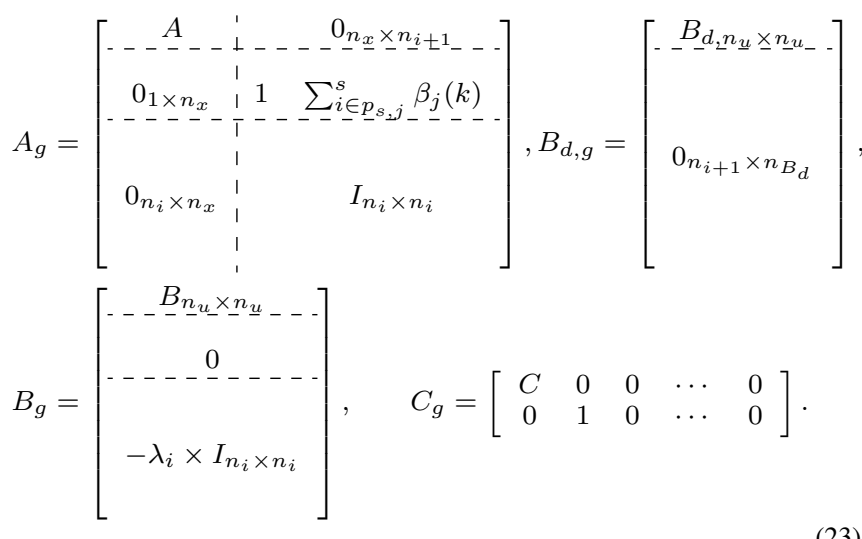

Considering the control action $u_{i}(k)$ as the scheduling variable related to each actuator and state in the augmented MPC model, it can be considered (22) as an LPV model. In this way, by following MPC-LPV approach in [10], the MPC optimization problem (7) can be formulated as a QP problem by using an estimation of scheduling variables. That means the scheduling variables in the prediction horizon are estimated using the values from the previous MPC interation and applied to update the model matrices of the MPC controller. Indeed, the sequence of the control input is utilized to change the model matrices used in the prediction horizon. Therefore, according to [10], the cost function (6) with the new additional objective that aims to increase the system reliability can be revised in vector form as follows

$$
\min _{\mathbf{u}(k), \mathbf{x}(k), \boldsymbol{\xi}(k), Q_{g}} \sum_{l=0}^{N_{p}}\left[\ell_{e}(k)+\ell_{s}(k)+\ell_{\Delta u}(k)-\ell_{R g}(k)\right],
$$

subject to:

$$
\begin{aligned}
& \tilde{\mathbf{x}}(k)=\mathcal{A}(\Theta(k)) x(k)+\mathcal{B}(\Theta(k)) \mathbf{u}(k)+B_{r, d} d_{m}(k), \\
& 0=E_{u} u(l \mid k)+E_{d} d_{m}(k) \\
& x(l+1 \mid k) \geq x_{s}-\xi(l \mid k) \\
& \log Q_{g}(l+1 \mid k)=\tilde{\mathbf{x}}_{n x+1}(l \mid k) \\
& u(l \mid k) \in \mathbb{U}, \quad x(l \mid k), \in \mathbb{X} \\
& \xi(l \mid k) \geq 0, \quad x(0 \mid k)=x(k) .
\end{aligned}
$$

where $\ell_{R g}(k) \triangleq Q_{g}^{\top} w_{3} Q_{g}$ is additional objective with the corresponding weight $w_{3}$ into the EMPC-LPV cost function to maximize the system reliability.The optimization problem is solved as a QP problem according to that the predicted states $\Theta(k)$ are linear. 


\section{B. Including demand uncertainty using chance constraints}

The constraints satisfaction (7) can not be guaranteed unless uncertainty it is not explicitly considering in some way. Hence, the original constraints that include stochastic elements (2a) will formulated by means of probabilistic statements using the chance constraints framework (8). Then, form of a state joint chance constraint is described as

$$
\mathbb{P}\left[G(r) x \leq g(r), \forall r \in \mathbb{Z}_{\left[1, m_{x}\right]}\right] \geq 1-\delta_{x},
$$

where $\delta_{x} \in(0,1)$ is the risk acceptability level of constraint violation for the states, $G(r)$ and $g(r)$ indicate the $r$-th row of $G$ and $g$, respectively. This entails that all rows $r$ have to be jointly satisfied with the probability $1-\delta_{x}$. Also, the form of a state individual chance constraint is described as

$$
\mathbb{P}[G(r) x \leq g(r),] \geq 1-\delta_{x}, \quad \forall r \in \mathbb{Z}_{\left[1, m_{x}\right]}
$$

which requires that each $r$-th row of the inequality has to be satisfied individually with the respective probability $1-$ $\delta_{x, r}$, where $\delta_{x, j} \in(0,1)$. Then, according to (13), the state constraints can be described as follows:

$$
\begin{aligned}
& \mathbb{P}[G(r) x \leq g(r)] \geq 1-\delta_{x, r}, \quad \forall r \in \mathbb{Z}_{\left[1, m_{x}\right]} \\
& \sum_{r=1}^{m_{x}} \delta_{x, r} \leq \delta_{x}, \quad 0 \leq \delta_{x, r} \leq 1
\end{aligned}
$$

and, as recommended in [11], specifying a constant and equal value of risk to each individual constraint, that is $\delta_{x, r}=$ $\delta_{x} / m_{x}$ for all $r \in \mathbb{Z}_{\left[1, m_{x}\right]}$, then (27b) is obtained.

By considering a known (or approximated) quasi-concave probabilistic distribution function for the stochastic disturbance in the dynamic model (1), it yields to

$$
\begin{aligned}
& \mathbb{P}[G(r) x(k+1) \leq g(r)] \geq 1-\delta_{x, r} \Leftrightarrow F_{G(r) B_{d} d_{m}(k)}(g(r) \\
& -G(r)(A x(k)+B u(k))) \geq 1-\delta_{x, r} \\
& \Leftrightarrow G(r)(A x(k)+B u(k)) \leq g(r)-F_{G(r) B_{d} d_{m}(k)}^{-1}\left(1-\delta_{x, r}\right),
\end{aligned}
$$

for all $r \in \mathbb{Z}_{\left[1, m_{x}\right]}$, where $F_{G(r) B_{d} d_{m}(k)}($.$) and$ $F_{G(r) B_{d} d_{m}(k)}^{-1}($.$) are the cumulative distribution and the left-$ quantile function of $G(r) B_{d} d_{m}(k)$, respectively. In this way, according to (27a), the safety stocks are optimally assigned and designed by the constraint back-off effect due to the term $F_{G(r) B_{d} d_{m}(k)}\left(1-\delta_{x, r}\right)$ in (25). Therefore, the original state constraint set $\mathbb{X}$ is adjusted by the effect of the $m_{x}$ deterministic equivalents in (28) and substituted by the stochastic feasibility set provided by

$$
\begin{aligned}
& \mathbb{X}_{s}(k):=\left\{x(k) \in \mathbb{R}^{n_{x}} \mid \exists u(k) \in \mathbb{U},\right. \text { such that } \\
& \quad G(r)(A x(k)+B u(k)) \leq g(r)-F_{G(r) B_{d} d_{m}(k)}^{-1}\left(1-\delta_{x, r}\right) \\
& \text { and } \left.\quad E_{u} u(k)+E_{d} \bar{d}(k)=0\right\}, \quad \forall r \in \mathbb{Z}_{1, m_{x}}
\end{aligned}
$$

where $\bar{d}(k)=\mathbb{E}\left[d_{m}\right]$ is the first moment of $d_{m}$ for all $k \in$ $\mathbb{Z}_{0 \geq 0}$. The set $\mathbb{X}_{s}(k)$ is convex when non-empty for all $\delta_{x, r} \in$ $(0,1)$ in most distribution functions, due to the convexity of $G(r) x(k+1) \leq g(r)$ and the log-concavity assumption of the distribution. For some particular distributions, e.g., Gaussian, convexity is preserved for $\delta_{x, r} \in(0,0.5]$ [7].

\section{Enhancing system reliability using chance constraints}

According to the Section V-A, component and system reliability model can be included in the EMPC controller model. Besides, (29) provides a new constraint set according to the deterministic equivalent (28). However, (29) does not consider the states related to the component and system reliability. Hence, it is necessary to modify the constraint set (29) with probabilistic statements based on the component and system reliability. In this way, the system reliability is formulated in terms of probabilistic constraints as follows

$$
x_{R g}(k) \in\left\{x_{R g} \in \mathbb{R}^{n_{R}} \mid \mathbb{P}\left[G_{R g} x_{R g} \geq g_{R g}\right] \geq\left(1-\delta_{R g}\right)\right\}
$$

where $x_{R g}(k) \in \mathbb{R}^{n_{R g}}$ is system reliability state defined in (22), $\delta_{R g} \in(0,1)$ is the corresponding risk acceptability level of constraint violation. According to the above discussion and the effect of stochastic reliability in the model (22), (30) can be rewritten as

$$
\begin{aligned}
& \mathbb{P}\left[G_{R g} x_{R g}(k+1) \geq g_{R g}\right] \geq\left(1-\delta_{R g}\right) \Leftrightarrow F_{G_{R g}}\left(g_{R g}\right. \\
& \left.-G_{R g} x_{R g}(k+1)\right) \geq 1-\delta_{R g} \Leftrightarrow G_{R g} x_{R g}(k+1) \\
& \geq g_{R g}+F_{G_{R g} \eta}^{-1}\left(1-\delta_{R g}\right),
\end{aligned}
$$

where $\eta$ is a random vector whose components follow a normal distribution, $F_{G_{R g} \eta}($.$) and F_{G_{R g} \eta}^{-1}($.$) are the cumulative$ distribution and the left-quantile functions involved in the state-and actuator-health deterministic equivalent constraints, respectively. Hence, a preventive strategy can be performed to increase overall system reliability by guaranteeing that the system reliability at each time instant to remain above a safe threshold until a predefined maintenance horizon is reached. Then, the (31) can be formulated

$$
\begin{aligned}
& G_{R g} x_{R g}\left(k+N_{p} \mid k\right) \geq g_{R g}(k)+F_{G_{R g} \eta}^{-1}\left(1-\delta_{R g}\right), \\
& g_{R g}(k)=x_{R g, \min }(k):=x_{R g}(k)+N_{p} \frac{R_{\text {tresh }}-x_{R g}(k)}{k_{M}+N_{p}+k},
\end{aligned}
$$

where $x_{R g, \min }(k) \in \mathbb{R}^{n_{R g}}$ is the vector of minimum reliability of the system allowed for time instant $k$ and $R_{\text {tresh }} \in \mathbb{R}^{n_{R g}}$ is the vector of threshold for the terminal system reliability at a maintenance horizon $k_{M} \in \mathbb{Z}_{\geq 0}$. The right-hand side of (32b) is an identical restricting of the remaining allowable system reliability $\left(R_{\text {tresh }}-x_{R g}(k)\right)$ that is updated at each time step according to the applied control actions and guarantees that $x_{R g}(k) \geq R_{\text {tresh }}$ for $k=k_{M}$.

\section{Chance-constraints reliability-aware EMPC-LPV refor- mulation}

After the inclusion system reliability in the control law as an additional state of the control model the setting of the proposed economic reliability-aware MPC-LPV controller including deterministic equivalent constraints is presented. In this way, for given sequence of demands $d$, predicted system reliability $R_{g}$, acceptable risk levels $\delta_{x}$ and $\delta_{R g}$, the optimization problem associated with the deterministic equivalent for considered transportation DWN at each time step $k$ is expressed as follows:

$$
\min _{\mathbf{u}(k), \boldsymbol{\xi}(k), \mathbf{x}(k) \mathbf{x}_{\mathbf{R g}}(k)} \sum_{k=0}^{N_{p}}\left[\ell_{e}(k)+\ell_{s}(k)+\ell_{\Delta u}(k)-\ell_{R g}(k)\right],
$$




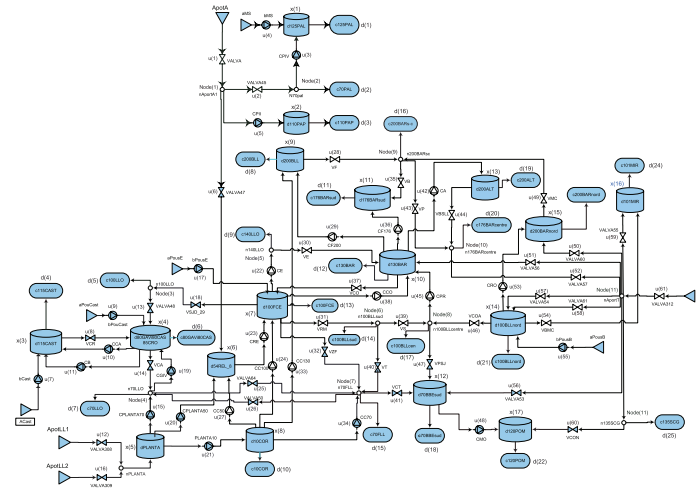

Fig. 1: Barcelona drinking water network.

subject to:

$$
\begin{aligned}
& \tilde{\mathbf{x}}(k)=\mathcal{A}(\Theta(k)) x(k)+\mathcal{B}(\Theta(k)) \mathbf{u}(k)+B_{r, d} d_{m}(k), \\
& 0=E_{u} u(l \mid k)+E_{d} d_{m}(k), \\
& x(k+l+1 \mid k) \leq x_{\max }(r)-\Phi_{k, r}^{x}\left(\delta_{x}\right), \\
& x(k+l+1 \mid k) \geq x_{\min }(r)+\Phi_{k, r}^{x}\left(\delta_{x}\right) \\
& G_{R g} x_{R g}\left(k+N_{p} \mid k\right) \geq x_{R g, \min }(k)+\Phi_{k, \eta}^{x_{R g}}\left(\delta_{R g}\right), \\
& x(k+l+1 \mid k) \geq x_{s}-\xi(k+l \mid k), \\
& \xi(k+l \mid k) \geq 0, \quad x_{R g}(l+1 \mid k)=R_{g}(k), \\
& \left.u(l \mid k) \in \mathbb{U}, \quad x(k \mid k), \bar{d}_{m}(k \mid k)\right)=\left(x(k), d_{m}(k)\right),
\end{aligned}
$$

for all $l \in \mathbb{Z}_{\left[0, N_{p}-1\right]}$ and all $r \in \mathbb{Z}_{\left[0, m_{r}\right]}$, where the terms $\Phi_{k, r}^{x}\left(\delta_{x}\right)=F_{G(r) B_{d} d_{m}(k)}^{-1}\left(1-\frac{\delta_{x}}{n_{x} N_{p}}\right)$ and $\Phi_{k, \eta}^{x_{R g}}\left(\delta_{R g}\right)=$ $F_{G_{R g} \eta}^{-1}\left(1-\frac{\delta_{x}}{N_{p}}\right)$ are the quantile functions involved in the states and system reliability deterministic equivalent constraints.

\section{APPLICATION}

The part of the Barcelona water transport network consists of 9 sources, 17 tanks and 61 actuators (valves and pumps), 12 nodes and 25 demands (see Figure 1). According to the DWN reliability study, demands, sources, pipelines and tanks are considered completely reliable whereas active elements such as valves and pumps are recognized not completely reliable [10]. According to the reliability analysis, it could be determined which states are structurally controllable since the path computation analysis gives all possible paths from a source to a target node. Furthermore, an approximate operational cost (according to the electricity cost of each element) and a maximal water flow (according to the physical constraints of the actuators) can be obtained for each path.

The objective of the MPC as has been explained before is to minimize the multi-objective cost function (33). The prediction horizon is 24 hours because the demand and the electrical tariff have periodicity of 1 day. The analysis is carried for a time period of 11 day ( 264 hours) with sampling time of 1 hour. The weights of the cost function (33a) are $W_{e}=100, W_{s}=1, W_{\Delta_{u}}=1$ and $W_{R_{g}}=10$.

In order to analyze and assess the benefits of the proposed economic readability-aware MPC-LPV approach, a comparison with respect to baseline control strategies that earlier proposed for the same case study is considered. In particular, the considered methods are the following:
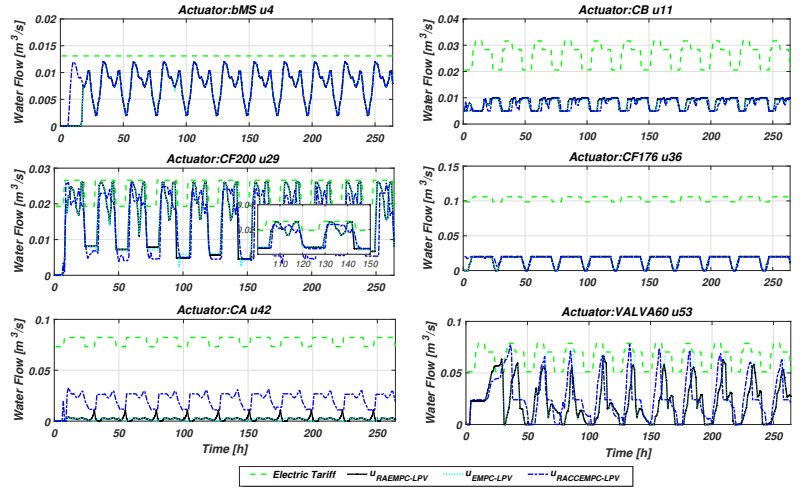

Fig. 2: Evaluation of the control actions results.

- Reliability-Aware Chance-constrained Economic MPC$L P V(R A C C E M P C-L P V)$ : This is the approach proposed in this paper that is based on solving the optimization problem (33). This approach allows to conisder nonstationary stochastic demand uncertainty and stochastic whole reliability of the system.

- Economic MPC-LPV (EMPC-LPV): This approach is based the optimization problem (33) without including the reliability objective. It is not considering the stochastic demand uncertainty, chance-constraints and terminal constraint of the system reliability of the network.

- Reliability-aware economic MPC-LPV (RAEMPC$L P V)$ : This approach relies on solving problem (24a). In this approach, an additional goal is included to the controller in order to extend the components and system reliability.

Table I exhibits the numeric assessment of the abovementioned controllers through different key performance indicators (KPIs), which are detailed below:

$$
\begin{aligned}
& K P I_{e}:=\frac{1}{n_{s}+1} \sum_{k=0}^{n_{s}} \alpha^{\top}(k) u_{k} \Delta_{t}, \\
& K P I_{\Delta_{u}}:=\frac{1}{n_{s}+1} \sum_{i=1}^{n_{u}} \sum_{k=0}^{n_{s}}\left(\Delta_{u}(i, k)\right)^{2}, \\
& K P I_{s}:=\sum_{i=1}^{n_{x}} \sum_{k=0}^{n_{s}} \max \left\{0, x_{s}(i, k)-x(i, k)\right\}, \\
& K P I_{R}:=x_{R g}(k), \\
& K P I_{t}:=t_{\text {opt }}(k),
\end{aligned}
$$

where $K P I_{e}$ denotes the average economic performance of the water network, $K P I_{\Delta_{u}}$ evaluates the smoothness of the control actions, $K P I_{s}$ comprises the quantity of water utilized from safety stocks, $K P I_{R}$ denotes the value of the whole system reliability of the DWN and $K P I_{t}$ defines the difficulty to solve the optimization tasks associated with each approach accounting $t_{o p t}(k)$ as the average time that gets to solve the corresponding FHOP. In $K P I_{e}, K P I_{\Delta_{u}}, K P I_{s}$ and $K P I_{t}$ lower values and $K P I_{R}$ higher value signify better performance results.

Figures 2 and 3 shows respectively the evolution of the valves and pumps commands and tank volumes for comparison of different considered MPC approaches for DWN. Figure 2 shows that pumps always try to operate at the minimum 
cost, i.e., when the electrical tariff is cheaper. Figure 3 shows the proper replenishment planning that the predictive controller dictates according to the cyclic behavior of demands. Although the behavior of the control inputs (valve and pump commands) (see Figure 2) and selected storage tanks (see Figure 3) are very similar in all the approaches, Figure 4 shows comparison of the system reliability predictions and accumulated economic cost of the DWN that obtained from the different MPC approaches. According to these results and reviewing the results in Tables I and II, it can be observed that the robustness enhancements of the RACCEMPC-LPV approach are larger than the other controllers in terms of reliability. The EMPC-LPV controller has lower values in the economic index $K P I_{e}$ but, the guarantee of reliability, robustness and feasibility problems are not considered. The main disadvantage of this controller is that control actions are computed based on economic criteria. In this cases the controller overexploits those actuators that have lower operational costs, quickening their damage and hazarding the service reliability. The RAEMPC-LPV strategy reached the lowest $K P I_{e}$ after the EMPC-LPV controller by including the reliability objective in the control low. However, the stochastic demand uncertainty and stochastic uncertainty of the system reliability are not considered.

Generally, the proposed RACCEMPC-LPV approach leads to a higher total closed-loop operational cost if considering
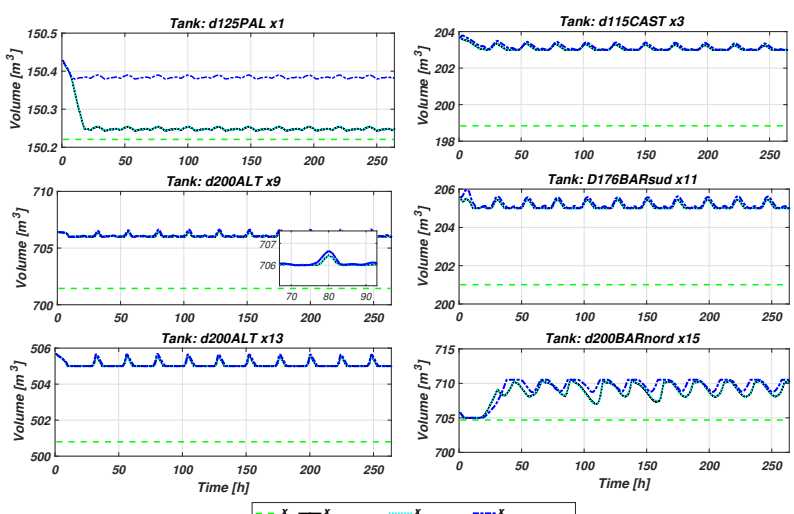

Fig. 3: Results of the evolutions of storage tanks.

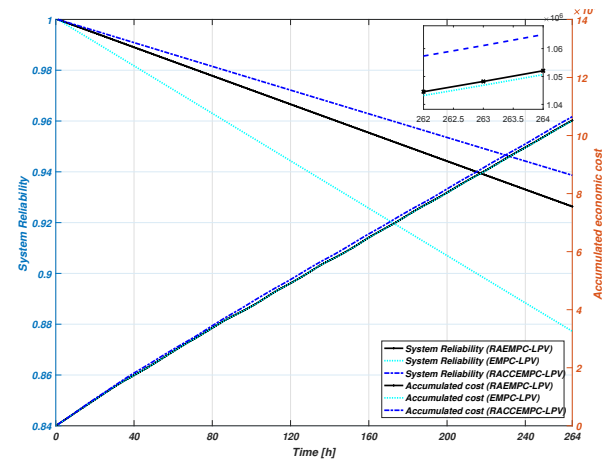

Fig. 4: Evaluation of system reliability and accumulated economic cost. only the water and electric costs as signs for economic performance. This is the price to pay for increasing the reliability of the system.

TABLE I: Comparison of control performance.

\begin{tabular}{lcccccc}
\hline Controller & $K P I_{e}$ & $K P I_{\Delta_{u}}$ & $K P I_{s}$ & $K P I_{R g}$ & $K P I_{t}$ & Simulation Time \\
\hline EMPC-LPV & 3779.81 & 0.5271 & 28951.72 & 0.8772 & 1.5628 & 412.599 \\
RAEMPC-LPV & 3980.07 & 0.5317 & 28952.62 & 0.9263 & 1.78348 & 470.841 \\
RACCEMPC-LPV & 4029.19 & 0.4903 & 28955.90 & 0.9386 & 1.9664 & 519.147 \\
\hline
\end{tabular}

TABLE II: Comparison of daily average costs of the MPC approaches.

\begin{tabular}{lccc}
\hline MPC Approach & $\begin{array}{c}\text { Water Average Cost } \\
\text { (e.u./day) }\end{array}$ & $\begin{array}{c}\text { Electric Average Cost } \\
\text { (e.u./day) }\end{array}$ & $\begin{array}{c}\text { Daily Average Cost } \\
\text { (e.u./day) }\end{array}$ \\
\hline EMPC-LPV & 44162.44 & 3053.08 & 47215.53 \\
RAEMPC-LPV & 44369.90 & 3121.84 & 47491.75 \\
RACCEMPC-LPV & 51438.13 & 3262.64 & 54700.77 \\
\hline
\end{tabular}

\section{CONCLUSION}

In this paper, an economic reliability-aware MPC-LPV strategy based on chance constraints for water transport network has been proposed to deal with the management of flow-based networks, considering both demand uncertainty and system reliability in a probabilistic way. By considering chance constraints programming to compute an optimal lreplenishment policy based on a desired risk acceptability level, the system reliability is introduced as state variables inside the control model, which includes nonlinear term and it is changed in a linear-like form through the LPV structure. The results obtained show that the system reliability of the DWN network is maximized with the proposed controller wile the cost increase. The level of resultant back-off volume is variable and depend of the forecast demand uncertainty and system reliability at each prediction step based on probabilistic distributions employed to their modelling.

\section{ACKNOWLEDGMENT}

This work has been partially funded by the Spanish State Research Agency (AEI) and the European Regional Development Fund (ERFD) through the projects DEOCS (ref. MINECO DPI2016-76493) and SCAV (ref. MINECO DPI2017-88403-R).

\section{REFERENCES}

[1] J. M. Maciejowski, Predictive control: with constraints, Pearson education, 2002.

[2] F. Karimi Pour, V. Puig, C. Ocampo-Martinez, Economic predictive control of a pasteurization plant using a linear parameter varying model, in: Computer Aided Chemical Engineering, Vol. 40, Elsevier, 2017, pp. 1573-1578.

[3] J. M. Grosso, C. Ocampo-Martínez, V. Puig, A service reliability model predictive control with dynamic safety stocks and actuators health monitoring for drinking water networks, in: Decision and Control (CDC), 2012 IEEE 51st Annual Conference on, IEEE, 2012, pp. $4568-4573$.

[4] G. C. Calafiore, F. Dabbene, R. Tempo, Research on probabilistic methods for control system design, Automatica 47 (7) (2011) 12791293. 
[5] G. Cembrano, J. Quevedo, M. Salamero, V. Puig, J. Figueras, J. Mart1, Optimal control of urban drainage systems. a case study, Control engineering practice 12 (1) (2004) 1-9.

[6] P. Kall, J. Mayer, et al., Stochastic linear programming, Vol. 7, Springer, 1976.

[7] J. M. Grosso, P. Velarde, C. Ocampo-Martinez, J. M. Maestre, V. Puig, Stochastic model predictive control approaches applied to drinking water networks, Optimal Control Applications and Methods 38 (4) (2017) 541-558.

[8] A. Shapiro, D. Dentcheva, A. Ruszczyński, Lectures on stochastic programming: modeling and theory, SIAM, 2009.

[9] G. B. Baecher, J. T. Christian, Reliability and statistics in geotechnical engineering, John Wiley \& Sons, 2005.

[10] F. Karimi Pour, V. Puig, G. Cembrano, Economic health-aware lpvmpc based on system reliability assessment for water transport network, Energies 12 (15) (2019) 3015.

[11] A. Nemirovski, A. Shapiro, Convex approximations of chance constrained programs, SIAM Journal on Optimization 17 (4) (2006) 969996. 